\title{
Les espaces domestiques à l'ère du cyberspace
}

Domestic Spaces in the Cyberspace Era

\section{Stéphanie Dadour}

\section{OpenEdition}

Journals

Édition électronique

URL : http://journals.openedition.org/crau/540

DOI : $10.4000 /$ crau. 540

ISSN : 2547-5746

\section{Éditeur}

Éditions du patrimoine

\section{Édition imprimée}

Date de publication : 1 novembre 2012

Pagination : $52-58$

ISBN : 978-2-7577-0108-9

ISSN : $1296-4077$

Référence électronique

Stéphanie Dadour, "Les espaces domestiques à l'ère du cyberspace», Les Cahiers de la recherche architecturale et urbaine [En ligne], 26/27 | 2012, mis en ligne le 01 novembre 2017, consulté le 24 avril 2019. URL : http://journals.openedition.org/crau/540; DOI : 10.4000/crau.540 
Cet article est un extrait $d$ 'un travail de thèse sur les espaces domestiques contemporains nordaméricains. II s'insère dans les débats qui traitent des limites entre les notions de privé et de public et cherche à proposer de nouvelles pistes de lectures des rapports qui structurent les espaces de l'habité à l'ère du cyberspace. II est en effet difficile aujourd'hui de discerner les frontières entre ces deux domaines. Les objets nomades, les médias, les nouvelles technologies et les réseaux investissent l'espace, modifient notre rapport à ce dernier, mais aussi au temps et à la sociabilité.

\section{Les espaces domestiques à l'ère du cyberspace}

Si, depuis le début du siècle dernier, I'histoire de l'habitation est fondée sur la différenciation entre les notions de public et de privé, du dehors et du dedans, de l'intérieur et de l'extérieur; et ce, à différents niveaux (spatial, politique, social, culturel et mental entres autres), force est de constater que ces rapports sont aujourd'hui ébranlés, d'autant plus, qu'au travers des ces dichotomies, se glisse la notion de l'intimité qui renvoie à celle de l'intériorité, fortement liée à la sphère privée, plus particulièrement à la maison et à la famille nucléaire, "clé de voûte ${ }^{1}$ » des sociétés occidentales en général. D'une part, les distinctions mentionnées sous-entendent une différentiation entre les notions mêmes et d'autre part, elles font référence à une séparation entre les entités binaires, difficiles à délimiter puisque l'ambiguïté de leurs frontières est le plus souvent liées à des constructions socio-historiques, à des modèles culturels et à leurs applications ${ }^{2}$.

La limite entre le privé et le public n'est pourtant pas figée dans le temps, ni dans l'action. Comme le rappellent Monique Eleb,

\footnotetext{
1. André Kaspi, François Durpaire,

Hélène Harter, Adrien Lherm,

La civilisation américaine, Paris,

PUF, 2004, p. 23.

2. Béatrice Collignon, Jean-François Staszak (dir.), Espaces domestiques.

Construire, habiter, représenter, Actes du colloque international sur les Espaces domestiques (17-20 septembre 2003, Institut de géographie de Paris), Paris, Bréal, 2003, p. 3.
} 
Anne-Marie Châtelet et Thierry Mandoul, les transformations des dispositifs spatiaux rendent compte des changements des modes de vie et de sociabilité : " La division de I'habitation en parties, en zones, qualifie à la fois le type de mode de vie qui y est préconisé et la conception des rapports entre les personnes. La structure du groupe domestique se lit à travers ces dissociations, ces positions, ces proximités ${ }^{3} »$. À des moments différents de l'histoire, ces notions étaient associées à des espaces et des aspects différents de ceux d'aujourd'hui, le domaine de l'espace domestique étant fortement ancrée et représentée par le privé et le dedans. En effet, Béatrice Collignon et Jean-François Staszak admettent que l'espace domestique ne se limite pas à un espace intérieur, qu'il « dépasse les murs, les fenêtres et les volets pour investir l'espace public, dans lequel il se niche parfois, et avec lequel il dialogue en permanence. Ce qui se passe à l'intérieur et la disposition même de cet intérieur ne se comprennent que dans leur rapport dialectique avec l'extérieur ${ }^{4}$ ». Cet article $s^{\prime}$ insère justement dans les débats qui traitent des limites entre les notions de privé et de public en ce qui concerne les espaces du domestique et cherche à proposer de nouvelles pistes de lectures des rapports qui structurent les espaces de l'habité à l'ère du cyberspace.

\section{Des espaces intermédiaires en voie d'être redéfinis}

En 1999, I'exposition The Un-Private House du commissaire Terence Riley qui s'est tenu au MoMA, à New York, pose les prémisses d'une nouvelle définition de l'espace domestique, désormais un-private ${ }^{5}$. Riley introduit les conséquences des évolutions culturelles, médiatiques, techniques et technologiques sur I'habitat et leurs rôles de catalyseur dans le champ de l'innovation et de l'expérimentation architecturale. II propose ainsi une lecture de la notion de privauté dans l'habitat et la définit dans son rapport à la maison. Le privé est alors compris comme la séparation des habitants de leurs activités dans l'espace public, et des autres maisons. Dans ce sens, la maison réunie et investit la famille pour l'exclure des activités extérieures et devient ainsi responsable de rituels et d'un confort propre à l'espace domestique ${ }^{6}$. Le privé, la famille et le travail sont les principales thématiques qu'il met en œuvre pour introduire l'idée, selon laquelle les technologies de communication d'aujourd'hui, représentatives de la sphère publique, pénètrent l'espace de la maison, le transforment et le rendent plus perméable.

Au-delà de la télévision et du téléphone, les nouvelles technologies reliées à Internet modifient les relations sociales et familiales et bouleversent les pratiques traditionnellement inscrites dans un cadre spatiotemporel fixe. Les limites sont brouillées par l'investissement d'objets nomades et la présence permanente des médias dans l'espace de la maison. Les objets nomades, notion introduite par Jacques Attali ${ }^{7}$ en 1985 , sont constitués de petites machines « miniaturisées capables de retenir, stocker, traiter, transmettre l'information - sons, images, données - à très grande vitesse » dont les deux outils majeurs sont le téléphone portable et Internet, tous deux interconnectés, et qui permettent aux nomades comme aux sédentaires, de se doter « d'une adresse non territoriale (numéro

\footnotetext{
3. Monique Eleb, Anne-Marie Châtelet,

Thierry Mandoul, Penser l'habité. Le

logement en questions, Liège, Mardaga,

1988, p. 61

4. Ibid. p. 8.

5. Le préfixe un exprime la notion

d'opposition.
}

6. Terence Riley, The Un-Private House, New York, MoMA, 1999, p. 9.

7. Jacques Attali, Une brève histoire de l'avenir, Paris, Fayard, 2006, p. 103. 
de téléphone mobile ou adresse électronique) » mais littéralement habitée. Dans cette perspective, le seuil, espace de transition par excellence, perd de sa capacité de passage d'une sphère à une autre puisque la maison n'est plus délimitée, ni détachée du public, ni confinée à un espace territorial mais qu'elle renvoie à un espace connecté en permanence avec l'extérieur. La maison, traditionnellement considérée du domaine de la sphère privée se voit entretenir un nouveau statut et se transformer pour conjuguer simultanément dimensions intimes et globales ${ }^{8}$.

De ce fait, si, comme le note Paul Claval'9, la télévision, nouvelle fenêtre virtuelle a modifié " les rapports entre espace domestique et espace extérieur » et "bouleversé les rapports du ménage qui l'entoure ", les ordinateurs et Internet réorganisent, eux aussi la maison, selon Eeva Jokinen ${ }^{10}$, et génèrent de nouveaux espaces publics et personnels à l'intérieur même de I'habitat. À travers une lecture des paradigmes sociaux ${ }^{11}$ qui transforment la maison et les activités qui y sont effectuées, Jokinen se réfère continuellement aux objets nomades multifonctionnels qui d'une part, facilitent la communication et confortent ainsi les individus dans le sens où ils contribuent à amplifier le sentiment de sécurité mais qui, autre part, rendent disponible leurs propriétaires en tout temps, les empêchant de se retrouver seuls, puisqu'ils sont continuellement mobilisés et interpellés.

Paradoxalement, dans le cadre de la maison, l'ordinateur incarne à la fois le seul espace personnel et privatif des individus mais aussi celui qui configure une hybridité entre l'individu, la machine et les espaces. La multifonctionnalité des objets nomades, la disparition d'espaces unifonctionnels et la disponibilité continuelle de leurs usagers distinguent les catégories des principes modernistes inscrits dans le temps, de l'espace et I'action ${ }^{12}$ où rien n'a une place assignée ou une fonction propre : le téléphone portable permet d'envoyer des messages, de prendre des photos, de se connecter sur Internet, de stocker de la musique, etc. De même, les espaces domestiques se vendent, se créent et s'organisent autour de la formulation de scénarios de vie. À la manière d'Ikea, les espaces ne sont plus destinés à un usage ou à une fonction précise. Les pratiques des habitants transforment les espaces au gré du travail à effectuer et du temps alloué. Ces espaces sont le plus souvent flexibles en termes d'activités et leurs statuts, tout comme leurs nominations deviennent ambiguës. Ces dénominations sont d'autant plus difficiles à cerner puisque l'aller et retour continuel entre le réel et le virtuel traduit la difficulté de catégoriser les espaces: la pièce dans laquelle l'individu se situe n'est pas proprement conçue pour l'activité qui s'y déroule et loin de correspondre aux espaces virtuels et/ou médiatiques auxquels il est connecté.

Le modèle du Home Sweet Home perd ses valeurs dichotomiques tout comme Haraway y introduit une nouvelle identité, celle de la citoyenneté cyborg, créature qui tisse la toile ${ }^{13}$ : "En imaginant qu'il fut un jour idéologiquement possible de définir la vie des femmes par la distinction entre les domaines privés et publics - ce que suggèrent les images d'une vie divisée entre l'usine et la maison pour la classe laborieuse, entre le marché et la maison pour la bourgeoisie, et entre
8. Hanna Johansson, Kirsi Saarikangas, (dir.), Homes in Transformation: Dwelling, Moving, Belonging, Helsinki, Suomalaisen Kirjallisuuden Seura, 2009, p. 13.

9. Paul Claval, «Les ouvertures de l'espace domestique ", dans Espaces domestiques. Construire, habiter, représenter, op. cit. note 2, p. 74

10. Eeva Jokinen, "Home, Work and Affects in the Fourth Shift », dans Homes in
Transformation..., op. cit. note 8, p. 358.

11. En référence aux trois stades du capitalisme liés à des formes spécifiques de familles introduits par Donna Haraway dans le Manifeste cyborg (à partir de l'équipe de recherche composée de Jussi Vähämäki, Mikko Jakonen, Leena Åkerblad et elle-même) auxquels elle ajoute un quatrième niveau marqué par l'enchevêtrement des espaces de travail et de la maison, et donc de la famille.
12. Sigfried Giedion, Space, Time and Architecture, Cambridge (Mass.), Harvard University Press, 1978.

13. Donna Haraway, Manifeste cyborg et autres essais, Paris, Exils, 2007, p. 63. 
le personnel et le politique selon le genre - une telle idéologie est maintenant totalement fallacieuse, et elle ne réussit même pas à montrer comment chaque terme de ces dichotomies construit l'autre en pratique et en théorie. Je lui préfère l'image idéologique du réseau, qui suggère une profusion d'espaces et d'identités, et une perméabilité des frontières du corps personnel et du corps politique ${ }^{14}$ ». En effet, Donna Haraway suggère dans ce sens, de repenser les binarités ${ }^{15}$ qui régissent nos systèmes en de nouveaux concepts et termes, en brouillant leurs frontières à travers de nouvelles figures d'écriture, de discours et de représentations et en introduisant l'identité cyborgienne, amoderne, et qu'elle relie au concept de femme de couleur, non-homme, non-blanche ${ }^{16}$.

Au-delà de ces ambiguïtés de frontières, Bruno Queysanne, dans sa présentation au colloque Autour de I'Étrangeté ${ }^{17}$, discute du seuil, signifié par la porte, pour exprimer la manière dont le dehors se transforme en dedans ou plus particulièrement de cet espace du seuil qui a pour fonction de transformer l'étranger en invité ou de confirmer l'amicalité de l'étranger. Dans le même sens, Paul Claval explique que la porte, cette ouverture passagère, une fois refermée, coupe l'espace domestique du monde, ce qui conforte physiquement et psychologiquement ${ }^{18}$. Symbole de franchissement d'une limite, en l'occurrence celle qui permet le passage du privé au public, du dehors au dedans, le seuil semble unidirectionnel : la notion de seuil est rarement employée pour désigner l'entrée dans la sphère publique mais le plus souvent pour indiquer le passage à l'intime et conférer un sentiment de sécurité. Queysanne remet en question les propos émis par Bruno Latour lors de sa présentation, qui dissolvait à partir d'une lecture du philosophe allemand Peter Sloterdijk la notion de l'extérieur pour n'évoquer que celle d'un intérieur toujours pris dans un autre intérieur, dans une sphère. II insiste ainsi sur l'importance de la différenciation entre extérieur/intérieur pour rappeler la capacité du seuil (et des espaces de transition) à modifier le statut d'autrui, celui de l'appartenance à, de l'intérieur et de la différence de l'extérieur, réaffirmée par Johansson et Saarikangas : «Ces pôles opposés distinguent les éléments du domaine du familier à ceux de l'étranger en établissant des frontières entre le "nous" et les "autres" [...] l'émergence de la notion moderne de maison a signifié la séparation de la sécurité, du confort, et de la propreté de la maison par rapport au monde extérieur dangereux ${ }^{19}$ ».

Dans le même sens, Françoise Paul-Lévy et Marion Ségaud soulignent l'importance des délimitations et des hiérarchies : "Quoi de l'espace, dans l'espace ou comment l'espace est-il détenteur ou vecteur de la garantie d'identité, et que savons-nous qui permette de répondre? Dans la mesure où le dispositif symbolique ne peut fonctionner sans la notion de discontinu, de limite, et dans la mesure où toute organisation spatiale requiert la discontinuité, l'usage et le jeu des limites, on propose de considérer la délimitation comme un élément fondamental dans la constitution et la représentation des systèmes sociaux des sociétés. Les limites dessinent comme une nomenclature d'espaces différenciés dont on propose qu'ils puissent et doivent être tenus pour qualitativement différents. Certaines limites sont matériellement codées, d'autres naissent
14. Ibid. p. 63.

15. Il s'agit des binarités hiérarchique et organique qui président la tradition (patriarcale, colonialiste et capitaliste) occidentale des sciences et de la politique.

16. Ce concept se veut indifférent au genre et à la race.

17. "Autour de l'étrangeté. Une rencontre entre architecture et politique » est un colloque qui a eu lieu les 3 et 4 juin 2010 à Sciences-Po et à l'école nationale supérieure d'architecture de Paris-Malaquais.

18. P. Claval, «Les ouvertures de l'espace domestique », op. cit. note 9, p. 65.
19. H. Johansson, K. Saarikangas, (dir.), Homes in Transformation..., op. cit. note 8, p. 17. 
par le passage d'un type d'espace matériel à un autre, d'autres sont à peine marquées matériellement, franches ou floues ${ }^{20} »$.

Ainsi, ces clivages ne se veulent pas définitifs et dichotomiques mais s'articulent matériellement, concrètement, en suivant une hiérarchie d'espaces dits intermédiaires ${ }^{21}$ et se caractérisent par un enchaînement transitionnel qui rappellent les rites de passage tel que définis par Victor Turner et introduits par Christian Moley : "La limite entre deux espaces opposés ne se réduit pas au rôle de séparation d'une simple frontière ou paroi ; elle appelle, dans les pratiques comme dans les dispositifs spatiaux, un franchissement graduel et contrôlé ${ }^{22}$ » que notre contemporanéité a ancré dans un système prévoyant, la résidentialisation. Celle-ci vise à contrôler de plus en plus (à la fois les espaces publics, intermédiaires que privés) pour la promotion (d'un semblant) de sécurité. Ceci est perceptible autant dans notre environnement bâti (codes de sécurité, clôtures) que dans nos activités quotidiennes (caméra de surveillance, cartes à puce). En effet, la résidentialisation, tente d'effacer l'ambiguïté entre les espaces à la recherche d'une clarification des statuts aux niveaux spatial, social et de la gestion où les espaces ouverts au grand public sont différenciés de ceux qui appartiennent aux résidents.

\section{Des espaces indéfinis à la fragilisation des liens sociaux}

À ces espaces en quête de définitions, s'ajoute aujourd'hui le processus d'individuation, au sens donné par François Ascher, qui est à la base d'une individua- tion de pratiques et d'une autonomie personnelle qui donne plus de liberté dans la gestion du temps et du choix des relations sociales de chacun. L'individuation des modes de vie consiste à permettre à chaque individu une autonomie et un horaire d'activité différent, renforcée par l'acquisition des objets nomades facilitant la connexion à des réseaux divers. Ainsi, les séparations traditionnelles qui se rapportent à des aspects spatio-temporels sont redéfinies au point de modifier les rapports sociaux façonnés par un isolement accru et un nouveau type d'intimité qui caractérisent la relation entre les individus et leurs objets nomades. Dolores Hayden y voit une situation dystopique où le paysage serait couvert de maisons électroniques (pour reprendre son expression Electronic Cottage) sans liens sociaux et voisinage ${ }^{23}$.

De manière paradoxale, cette souplesse spatiotemporelle transforme les rapports des individus à leur environnement. L'accessibilité aux mobilités réelles et virtuelles exprime une certaine liberté puisqu'elle permet de sortir des espaces familiers, de la maison en l'occurrence, à la recherche d'espaces d'affinités. Aux nombreuses institutions hiérarchiques auxquelles l'individu prend part, il est aujourd'hui possible de choisir et d'appartenir à celles qui s'apparentent le plus à ses propres intérêts. Dans cette perspective, l'identité sociale se caractérise par le choix, celui-ci étant facilité par la mobilité et par l'accès à un monde de réseaux sociaux sans contact réel. La notion de la force des liens faibles introduite par Ascher se définit par la multiplication des liens sociaux et par la diversification de la nature de ces liens qui " s'appuient sur des modes
20. Françoise Paul-Lévy, Marion Segaud, Anthropologie de l'espace, Paris, Centre Georges Pompidou, Centre de Création industrielle, 1983.

21. Christian Moley, Les abords du chez-soi. En quête d'espaces intermédiaires, Paris, Éditions de la Villette, 2006, p. 6.

22. Ibid, p. 9
23. Dolores Hayden, Building Suburbia, Green Fields and Urban Growth 1820-2000, New York, Vintage Books, 2003, p. 225. 
de communication multiples : échanges de messages sur Internet et se rencontrer au café sont en effet des interactions qualitativement différentes. Les liens sont également beaucoup plus "faibles" qu'autrefois, plus fragiles aussi. En revanche, il est plus facile d'en produire de nouveaux ${ }^{24}$. " Ainsi se crée ce qu'il appelle la solidarité commutative ou la société hypertexte qui se traduit par l'adhésion à une diversité de réseaux liés entre eux. D'innombrables réseaux aux objectifs différents sont créés chaque année: ils permettent de renouer des liens rompus ou oubliés, de rencontrer des gens (virtuels et réels), de faire la promotion d'une personne, d'un groupe, d'une idéologie, de créer un réseau professionnel, politique ou social, etc. Dans le prolongement de ceux-ci, des espaces virtuels (littéralement) se développent et donnent lieu à des expériences potentiellement nouvelles qui constituent et qui interrogent de nombreuses pratiques.

Ces espaces en construction offrent, de par leur structure, leur fonctionnement, leur langage ${ }^{25}$ (lexique propre à chacun d'eux), leurs propres systèmes économique, politique et culturel, des possibilités exponentielles. L'expression culture numérique a, en effet, un impact aux niveaux sociologique et anthropologique sur les modes de $v^{2}{ }^{26}$. Dans ce sens, si les années 1990 étaient marquées par l'émergence de Homepages personnelles et de blogs qui représentent un chez-soi et la personnalité d'un individu, force est de constater, comme le souligne Susanna Paansonen, que les réseaux sociaux ont pris le dessus ${ }^{27}$.

Les réseaux sociaux tels que Facebook ou MySpace permettent aux individus de se représenter en fonction de leurs noms, de leur physique, de leurs activités, de leurs intérêts, de photos, de textes mais au-delà de ces détails (qui peuvent aussi être évités), ce sont la liste d'amis et l'adhésion à des groupes, c'est-àdire le réseau constitué qui définit la sociabilité et les possibles liens interactifs entre individus. Antoine Picon décèle au sein de cette cyber-sociabilité « de nombreux problèmes d'organisation spatiale » qui en effet, limitent les rencontres à la lecture de profils «au lieu de se mouvoir au sein d'espace pluridimensionnel permettant de visualiser des chaînes relationnelles plus longues ${ }^{28}$. " L'interface perd de son importance au profit du contenu à la différence du monde de Second Life par exemple, où la création d'un avatar paramétré, constitué manuellement et librement, est à la base des constructions sociales qui fonctionnent selon l'appartenance communautaire, la recherche d'affinités et l'environnement constitué.

Dans le monde de Second Life justement, il est question de scénarios, d'histoires qui se construisent et qui régissent l'environnement. Par ailleurs, les options qui permettent d'intégrer des images, de la musique, de la vidéo et des scripts altèrent l'espace, particulièrement son statut, et participent à sa transformation. Ainsi, la sociologue Régine Robin ${ }^{29}$ pointe du doigt la télévision, plus particulièrement les reality-shows, et I'Internet comme des vecteurs de l'expérimentation qui sont à la fois avantageux mais aussi dangereux dans la mesure où le vrai et le faux s'entremêlent et sont difficilement discernables constituant « un semblant d'extrême singularité dans une très grande uniformisation $^{30}$ » et qui sont à la base de la fragilisation du moi
24. François Ascher, Les nouveaux principes de l'urbanisme, Paris, Éditions de l'Aube, 2001 , p. 39

25. Comme le rapporte Susanna Paasonen, le vocabulaire et le langage qui les définit fait d'ailleurs allusion à la maison, au voyage et à la mobilité : Homepage, naviguer ou visiter sur Internet.
26. Antoine Picon, Culture numérique et architecture, une introduction, Bâle, Birkhäuser, 2010, p. 50

27. Susanna Paasonen, Immaterial Homes, Personal Spaces and the Internet as Rhetorical Terrain, dans H. Johansson, K. Saarikangas, (dir.), Homes in

Transformation..., op. cit. note 8, p. 348.
28. A. Picon, op. cit. note 26, p. 53.

19. Natalie Levisalles, « Il existe une tendance à emprunter n'importe quelle identité ", interview Régine Robin, Libération, 20 avril 2002. Consultable en ligne : http://www.liberation.fr/ week-end/0101410310-il-existe-unetendance-a-emprunter-n-importe-quelleidentite.

30. Ibid. 
dans la société contemporaine. L'entremêlement du réel et du virtuel définit la réalité de notre environnement et la constitution de dynamiques et de vocabulaires en construction. Au-delà du langage, c'est la signification et le sens donné à ces espaces qui déterminent leurs réceptions et leurs usages.

\section{Conclusion}

Enfin, si pendant longtemps les figures du seuil ont servi d'espaces intermédiaires et de transition entre le privé et le public, il est aujourd'hui d'une part, difficile de discerner les frontières entre ces deux domaines et d'autre part, compliqué, à l'intérieur même d'un espace, de comprendre les liens entre le réel et le virtuel. Les objets nomades, les médias, les nouvelles technologies et les réseaux investissent l'espace, modifient notre rapport à ce dernier, mais aussi au temps et à la sociabilité. Dans la continuité de cet article, il serait intéressant de saisir comment les architectes traduisent les notions de limites dans le contexte contemporain et quels sont les caractéristiques et le sens qui y sont prêtées? 